\title{
A missense mutation in MKRN3 in a Danish girl with central precocious puberty and her brother with early puberty
}

\author{
Johanna Känsäkoski ${ }^{1,2}$, Taneli Raivio ${ }^{1,2}$, Anders Juul ${ }^{3}$ and Johanna Tommiska ${ }^{1,2}$
}

BACKGROUND: Idiopathic central precocious puberty (ICPP) results from the premature reactivation of the hypothalamicpituitary-gonadal axis leading to development of secondary sexual characteristics prior to $8 \mathrm{y}$ in girls or $9 \mathrm{y}$ in boys. Since the initial discovery of mutations in the maternally imprinted MKRN3 gene in 2013, several case reports have described mutations in this gene in ICPP patients from different populations, highlighting the importance of MKRN3 as a regulator of pubertal onset.

METHODS: We screened 29 Danish girls with ICPP for mutations in MKRN3. Expression of MKRN3 in human hypothalamic complementary DNA (CDNA) was investigated by PCR.

RESULTS: One paternally inherited rare variant, c.1034G>A (p.Arg345His), was identified in one girl with ICPP and in her brother with early puberty. The variant is predicted to be deleterious by three different in silico prediction programs. Expression of MKRN3 was confirmed in adult human hypothalamus.

CONCLUSION: Our results are in line with previous studies in which paternally inherited MKRN3 mutations have been found both in males and in females with ICPP or early puberty. Our report further expands the set of MKRN3 mutations identified in ICPP patients across diverse populations, thus supporting the major regulatory function of MKRN3 in pubertal onset.

C entral precocious puberty $(\mathrm{CPP})$ results from the premature reactivation of the hypothalamic-pituitary-gonadal axis leading to the development of secondary sexual characteristics prior to the age of $8 \mathrm{y}$ in girls or $9 \mathrm{y}$ in boys (1). In cases of idiopathic CPP (ICPP), there is no anatomical abnormality which can be identified as an obvious cause for the premature onset of puberty, suggesting there might be a genetic cause. However, the genetic basis for control of pubertal onset remains largely unresolved. Further knowledge can be gained through identification of gene defects underlying disorders where the normal timing of puberty is disrupted, such as in ICPP. Several genes, including KISS1, KISSIR, GNRHR, LIN28B, LIN28A, GABRA1, NPY1R, TAC3, and TACR3, have been proposed as candidate genes in ICPP based on genetic linkage and/or function of the gene product, but conclusive evidence for disease-causing mutations in these genes has not been found (2).

Until the recent discovery of three truncating and one missense mutation in MKRN3 (3), only rare activating mutations in KISS1 and KISS1R, stimulating the secretion of gonadotropin-releasing hormone $(\mathrm{GnRH})$ from the hypothalamus, had been identified to underlie CPP $(4,5)$. The maternally imprinted MKRN3 gene is located on chromosome 15q11.2 in the Prader-Willi syndrome critical region and encodes Makorin RING-finger protein 3, a putative E3 ubiquitin ligase belonging to the Makorin family of zinc-finger proteins. The exact function of MKRN3 in the control of pubertal onset is still unknown but is presumed to be inhibitory to $\mathrm{GnRH}$ secretion based on the declining expression in the mouse arcuate nucleus before the onset of puberty (3). Since the initial discovery, there have been four other reports of paternally inherited mutations in MKRN3 in ICPP patients from different populations (6-9), providing further support for the significant contribution of mutations in this gene to the premature initiation of puberty. It is therefore well justified to screen this gene in ICPP patients regardless of ethnic background.

In this study, we screened MKRN3 in 29 Danish girls with ICPP who were previously not found to have causative mutations in KISS1, KISS1R, LIN28A, or LIN28B (10). Since the expression of MKRN3 in human has only been reported at the level of the whole brain (11), we also decided to investigate whether MKRN3 is expressed in an adult human hypothalamic cDNA library.

\section{RESULTS}

One heterozygous missense variant, c.1034G >A (p.Arg345His), in MKRN3 was found in one girl with ICPP and was subsequently identified also in her brother with early puberty. The variant is inherited from the father as the mother did not carry it, and it has been reported once in 4,300 individuals in the European American population in the NHLBI Exome Sequencing Project database (Exome Variant Server, NHLBI GO Exome Sequencing Project, Seattle, WA). The substitution of the highly conserved arginine at position 345 to histidine is predicted to be deleterious by all three prediction programs 
used: (PolyPhen-2: "probably damaging” (score: 1.000); SIFT: "affects protein function" (score 0.01); Mutation Taster: "disease causing" (probability 0.999999998)). No other rare variants were found in the 29 patients studied.

The index patient was a girl who was born for non-consanguinous Caucasian parents. She developed intermittent breast enlargement from 6 y of age and experienced rapidly progressing breast and pubic hair development from $7 \mathrm{y}$ of age. She was seen at $7.5 \mathrm{y}$ when she presented with breast stage 4 and pubic hair stage 3 . She had accelerated linear growth (height standard deviation score +2.0$)$, and bone age was advanced $(10 \mathrm{y}$ Greulich Pyle). A GnRH test ( $0.1 \mathrm{mg}$ of Relefact LH-releasing hormone) showed pubertal LH response (from 1.28 to 32.2 IU/l). Basal estradiol was $68 \mathrm{pmol} / \mathrm{l}$, inhibin B $124 \mathrm{pg} / \mathrm{ml}$, and IGF-I $469 \mathrm{ng} / \mathrm{ml}$ (2.82 SDS). Brain MRI was normal. She was treated with $\mathrm{GnRH}$ analogue for $2 \mathrm{y}$. After that, GnRH analogue was stopped, and puberty resumed and menarche occurred at $11.5 \mathrm{y}$ of age. Her final height was $164.2 \mathrm{~cm}(0.38$ SD) (target height $169.2 \mathrm{~cm}(-0.06 \mathrm{SD})$; Figure 1). Her mother had menarche at $11 \mathrm{y}$, puberty in father was reported as "average," and her brother had very early voice break at $10.5 \mathrm{y}$. He was seen at regular visits because of cystic fibrosis.

PCR and agarose gel electrophoresis revealed strong MKRN3 expression in the human hypothalamic cDNA library (Figure 1).

\section{DISCUSSION}

In this study, we identified a missense variant in MKRN3 with a very probable deleterious effect in a Danish girl with ICPP and her brother with early puberty. The variant is paternally inherited since it was present in both siblings but absent in their mother. The variant was also found in the European American population in the NHLBI Exome Sequencing Project database with a frequency of 1 per 8,600 chromosomes, so it is extremely rare. It is important to note that because of the maternal imprinting of $M K R N 3$, the effects of deleterious mutations become apparent only if they are inherited

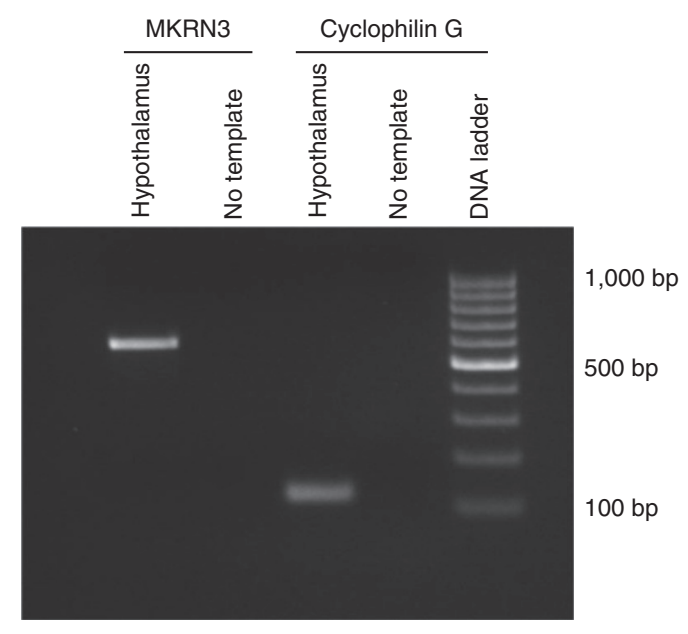

Figure 1. MKRN3 is expressed in the human hypothalamus. A 598-bp fragment of transcript encoding MKRN3 was amplified from the human hypothalamic cDNA library. Amplification of cyclophilin G was used as the housekeeping control gene. The PCR products were visualized on a $2.0 \%$ agarose gel. paternally, and these rare mutations can also be present in the global population. In addition, the timing of puberty of the mutation carrier in the database is unknown.

Although ICPP is typically inherited from mother to daughter and affects mostly girls (12), mutations in MKRN3 seem to be equally distributed among males and females, although the effect on females seems to be somewhat stronger $(3,6)$. In our family, the girl's age at the onset of puberty was comparable to previous reports in female $M K R N 3$ mutation carriers (range: 3.0-6.5 y; mean 5.9; median 6.0 y) (13). Although the boy's pubertal development was not formally assessed, his voice break occurred very early at $10.5 \mathrm{y}$ of age (14), suggesting his puberty was already advanced at that age (15).

Eight MKRN3 mutations described in ICPP patients so far have been mutations leading to an early stop codon, and only four missense mutations have been described previously. The p.Arg345His mutation identified in this study is situated in the C3HC4 RING domain of MKRN3 responsible for ubiquitin ligase activity of the protein. Two of the four previously reported missense mutations in ICPP patients (p.Arg365Ser and p.Cys340Gly) are situated in this same domain $(3,6)$, but the functional consequences of these mutations, although predicted to be deleterious and disrupt the tertiary protein structure (6), have not been confirmed. The two other reported missense mutations, p.Phe417Ile and p.His420Gln, are located in the third RNA-binding $\mathrm{C} 3 \mathrm{H}$ zinc-finger domain and are also predicted to have deleterious effects $(7,9)$. It will be highly interesting to test the effects of these mutations and the mechanism by which MKRN3 regulates the onset of puberty once a bioassay is available.

The expression of $M k r n 3$ was shown to decrease in the mouse arcuate nucleus at the same age as the expression of Kiss1 and Tac2 increase, suggesting Mkrn3 might act as a break for pubertal onset (3). MKRN3 has been reported to be ubiquitously expressed in human adult and fetal tissues (11); however, only in a few patients with MKRN3 mutations have additional phenotypic features been identified in addition to CPP $(3,7)$, suggesting at least partially redundant roles outside the hypothalamic-pituitary-gonadal axis. We also found MKRN3 to be expressed in the adult human hypothalamus, and the expression seems to be quite high in contrast to what was previously found in the mouse arcuate nucleus (3). The obvious limitation of our analysis is that, as human hypothalamic samples are poorly available, we were unable to study whether there is a change in the expression between different-aged individuals. However, the clear hypothalamic expression suggests that the proposed inhibitory regulation of GnRH expression and/ or release is only one of MKRN3's hypothalamic functions, occurring in a cell type-dependent or time-dependent manner. MKRN3 has both RNA-binding zinc-finger domains as well as an E3 ubiquitin ligase domain, indicating it has functions in regulation of gene expression, targeting proteins for degradation, and/or other modulation of protein function. Mutations in ICPP patients have been found both in the E3 ligase domain as well as in the third zinc finger domain, suggesting that both of these domains are important for the correct function of MKRN3 in the regulation of puberty. 
In conclusion, mutations in MKRN3 are the most frequent known genetic cause of ICPP to date in several different populations. We screened 29 Danish girls and identified one probably deleterious missense variant, which is about the same frequency as observed previously in 207 unrelated girls with ICPP from three different university medical centers ( 7$)(1 / 29=$ $3.4 \%$ vs. $8 / 207=3.9 \%)$. Our report further expands the set of MKRN3 mutations identified in ICPP patients across diverse populations, thus supporting the major regulatory function of MKRN3 in pubertal onset.

\section{METHODS}

\section{Subjects}

Twenty-nine girls with ICPP were recruited from our outpatient clinic at the Department of Growth and Reproduction, Copenhagen University Hospital, Denmark. Details of this study have previously been published (10).

\section{Molecular Genetic Analysis}

Genomic DNA from peripheral blood leukocytes of the subjects was extracted. The coding exon of MKRN3 was PCR amplified from all subjects with ICPP and from one patient's mother and brother. DNA from the patient's father was not available. PCR products were purified with ExoSAP-IT treatment (Amersham Biosciences, Piscataway, NJ) and sequenced using the ABI BigDyeTerminator Cycle Sequencing Kit (v3.0) and ABI 3730xl 96-capillary DNA Analyzer automated sequencer (Applied Biosystems, Foster City, CA). Sequences were aligned and read with Sequencher 4.9 software (Gene Codes, Ann Arbor, MI). All primer sequences and PCR conditions are available upon request.

The effects of the identified genetic variant were predicted in silico with three programs: PolyPhen-2, SIFT, and Mutation Taster.

\section{Expression in Adult Human Hypothalamus}

A 598-bp fragment of cDNA transcript encoding MKRN3 was amplified from human hypothalamic cDNA library (Clontech Laboratories, Mountain View, CA) constructed from the hypothalami of 30 male/ female Caucasians, aged 15-68 y, after sudden death.

\section{Ethics}

The study was approved by the scientific ethical committee for Copenhagen and Frederiksberg (\# KF 01282214 and KF 11 20062033), and the Danish Data Protection Agency. All participants and their parents gave informed consents.

\section{ACKNOWLEDGMENTS}

Lea Puhakka is thanked for skillful technical assistance.

\section{STATEMENT OF FINANCIAL SUPPORT}

This work was supported by the Academy of Finland, the Helsinki University Central Hospital Research Funds, Foundation for Pediatric Research
(Helsinki, Finland), the Danish Research Council (A.J.), and the Capital Region of Copenhagen (A.J.).

Disclosure: The authors declare that they have no competing interests.

\section{REFERENCES}

1. Parent AS, Teilmann G, Juul A, Skakkebaek NE, Toppari J, Bourguignon JP. The timing of normal puberty and the age limits of sexual precocity: variations around the world, secular trends, and changes after migration. Endocr Rev 2003;24:668-93.

2. Teles MG, Silveira LF, Tusset C, Latronico AC. New genetic factors implicated in human GnRH-dependent precocious puberty: the role of kisspeptin system. Mol Cell Endocrinol 2011;346:84-90.

3. Abreu AP, Dauber A, Macedo DB, et al. Central precocious puberty caused by mutations in the imprinted gene MKRN3. N Engl J Med 2013;368:246775.

4. Teles MG, Bianco SD, Brito VN, et al. A GPR54-activating mutation in a patient with central precocious puberty. N Engl J Med 2008;358:709-15.

5. Silveira LG, Noel SD, Silveira-Neto AP, et al. Mutations of the KISS1 gene in disorders of puberty. J Clin Endocrinol Metab 2010;95:2276-80.

6. Settas N, Dacou-Voutetakis C, Karantza M, Kanaka-Gantenbein C, Chrousos GP, Voutetakis A. Central precocious puberty in a girl and early puberty in her brother caused by a novel mutation in the MKRN3 gene. J Clin Endocrinol Metab 2014;99:E647-51.

7. Macedo DB, Abreu AP, Reis AC, et al. Central precocious puberty that appears to be sporadic caused by paternally inherited mutations in the imprinted gene makorin ring finger 3. J Clin Endocrinol Metab 2014;99:E1097-103.

8. Schreiner F, Gohlke B, Hamm M, Korsch E, Woelfle J. MKRN3 mutations in familial central precocious puberty. Horm Res Paediatr 2014;82: $122-6$.

9. de Vries L, Gat-Yablonski G, Dror N, Singer A, Phillip M. A novel MKRN3 missense mutation causing familial precocious puberty. Hum Reprod 2014;29:2838-43.

10. Tommiska J, Sørensen K, Aksglaede L, et al. LIN28B, LIN28A, KISS1, and KISS1R in idiopathic central precocious puberty. BMC Res Notes 2011;4:363.

11. Jong MT, Gray TA, Ji Y, et al. A novel imprinted gene, encoding a RING zinc-finger protein, and overlapping antisense transcript in the PraderWilli syndrome critical region. Hum Mol Genet 1999;8:783-93.

12. de Vries L, Kauschansky A, Shohat M, Phillip M. Familial central precocious puberty suggests autosomal dominant inheritance. J Clin Endocrinol Metab 2004;89:1794-800.

13. Macedo, DB, Brito, VN, Latronico, AC. New causes of central precocious puberty: the role of the genetic factors. Neuroendocrinology 2014; 100:1-8.

14. Juul A, Magnusdottir S, Scheike T, Prytz S, Skakkebaek NE. Age at voice break in Danish boys: effects of pre-pubertal body mass index and secular trend. Int J Androl 2007;30:537-42.

15. Harries ML, Walker JM, Williams DM, Hawkins S, Hughes IA. Changes in the male voice at puberty. Arch Dis Child 1997;77:445-7. 